\title{
Complex phases in the doped two-species bosonic Hubbard Model
}

\author{
Kalani Hettiarachchilage, ${ }^{1,2}$ Valéry G. Rousseau, ${ }^{1}$ Ka-Ming Tam,,${ }^{1,2}$ Mark Jarrell, ${ }^{1,2}$ and Juana Moreno ${ }^{1,2}$ \\ ${ }^{1}$ Department of Physics and Astronomy, Louisiana State University, Baton Rouge, Louisiana 70803, USA \\ ${ }^{2}$ Center for Computation and Technology, Louisiana State University, Baton Rouge, LA 70803, USA
}

(Dated: July 27, 2018)

\begin{abstract}
We study a doped two-dimensional bosonic Hubbard model with two hard-core species using quantum Monte Carlo simulations. With doping we find five distinct phases, including a normal liquid at higher temperature, an anti-ferromagnetically ordered Mott insulator, a region of coexistent anti-ferromagnetic and superfluid phases near half filling, and further away from half filling, a superfluid phase and a phase separated ferromagnet. In the latter, the heavy species has Mott behavior with integer fillings, while the light species shows Mott and superfluid behaviors. The global entropy of this phase is relatively high which may provide a new avenue to obtain a polarized phase or a Mott insulator in cold atom experiments.

PACS numbers: $02.70 . \mathrm{Uu}, 05.30 . \mathrm{Jp}$
\end{abstract}

Cold atoms experiments $\underline{\underline{1}}$ have become a playground for realizations of the Hubbard $2, \underline{3}$ and other strongly correlated model Hamiltonians, since model parameters can be tuned using laser and magnetic fields $\stackrel{4}{\underline{4} .}$ Recently, there has been an increasing interest in studies of mixtures of atoms 6.7 due to the complexity associated with multiple species and the possibility of discovering novel phases. The experimental study of the ${ }^{87} R b^{41} \mathrm{~K},{ }^{6} \mathrm{Li}$ ${ }^{40} \mathrm{~K}$ and different alkaline earth mixtures in an optical lattice $\underline{\underline{8}-10}$ have motivated theoretical studies of the Hubbard model with two species with different masses $\underline{11-15}$ These studies reveal a rich phase diagram at half-filling. Experimental studies of the two species Bose-Hubbard model shows that the massive species exhibits Mott while the other shows superfluid behavior $\stackrel{\underline{8}}{=}$ Since the carrier concentration can be controlled in experiments, we explore the doping dependence of the model to find complex and exotic phases 16 .

While experimental controllability is a remarkable aspect of atomic systems, the major goal of simulating quantum magnetism still remains a challenge. The main obstacle is reaching the low entropy and temperature required to observe magnetically ordered or Mott insulating phases. Various methods have been suggested in the last decade or so. $\stackrel{17-19}{-19}$ A recent proposal by Ho and Zhou suggests that the entropy of a Fermi gas can be squeezed into a surrounding Bose-Einstein condensed gas, which acts as a heat reservoir $\stackrel{20}{=}$ These light particles are then evaporated, leaving behind a low-entropy Fermi gas.

In this Rapid Communication, we show that the two species bosonic Hubbard model with a mass imbalance at finite doping exhibits a ferromagnetic phase separated state, in addition to superfluid and antiferromagnetic phases. This state has entropy similar to the superfluid indicating that it should have similar experimental accessibility. Furthermore, it exhibits the entropy squeezing phenomenon in which the heavy particles form a Mott insulating phase, while the light ones are in a superfluid phase that act as a heat reservoir to absorb entropy. In addition, we study the entire phase diagram of this two species bosonic Hubbard model as a function of temper- ature and doping.

Our study is based on the two-species Hubbard model with hard-core bosons $a$ and $b$ confined to a twodimensional lattice. The Hamiltonian takes the form:

$$
\begin{aligned}
\hat{\mathcal{H}}= & -t_{a} \sum_{\langle i, j\rangle}\left(a_{i}^{\dagger} a_{j}+H . c .\right) \\
& -t_{b} \sum_{\langle i, j\rangle}\left(b_{i}^{\dagger} b_{j}+H . c .\right)+U^{a b} \sum_{i} n_{i}^{a} n_{i}^{b},
\end{aligned}
$$

where $a_{i}^{\dagger}\left(b_{i}^{\dagger}\right)$ and $a_{i}\left(b_{i}\right)$ are the creation and annihilation operators, respectively, of hard-core bosons $a(b)$, with number operators $n_{i}^{a}=a_{i}^{\dagger} a_{i}, n_{i}^{b}=b_{i}^{\dagger} b_{i}$. The sum $\sum_{\langle i, j\rangle}$ runs over all distinct pairs of first neighboring sites $i$ and $j, t_{a}\left(t_{b}\right)$ is the hopping integral between $i$ and $j$ sites for species $a(b)$, and $U^{a b}$ is the strength of the interspecies repulsion. In the hard-core limit, the creation and annihilation operators satisfy commutation rules on different sites and anti-commutation rules on identical ones.

We perform quantum Monte Carlo simulations using the Stochastic Green Function algorithm 21 with global space-time updates ${ }^{22}$ for the canonical ensemble on $L \times$ $L$ lattices. We use an inverse temperature $\beta=8 L$ to capture the ground state properties. Our results at halffilling reproduce the phase diagram of Ref. 12. We focus on the unpolarized phase diagram, so our total density is $\rho=N / L$ with $N=N_{a}+N_{b}=2 N_{a}$, with $N_{a}$ and $N_{b}$ the number of heavy $a$ and light $b$ particles, respectively. We restrict our simulation to the following parameters corresponding to the strongly $\mathrm{AF}$ region at half filling: $t_{a}=0.08 t, t_{b}=t$, and $U^{a b}=6 t$, where $t=1$.

Fig. 1 displays signatures of ordering. To look for phase separation and Mott states, we calculate the chemical potential by adding one $a$ and one $b$ particle to the system as $\mu=(E(N+2)-E(N)) / 2$. The superfluid $(\mathrm{SF})$ phase is detected by measuring the superfluid density, $\rho_{S F}$, using the fluctuations of the winding number, $W$, via Pollock and Ceperley's formula 23 . We measure the correlated winding: $\left\langle W^{2}\right\rangle=\left\langle\left(W_{a}+W_{b}\right)^{2}\right\rangle$, where 

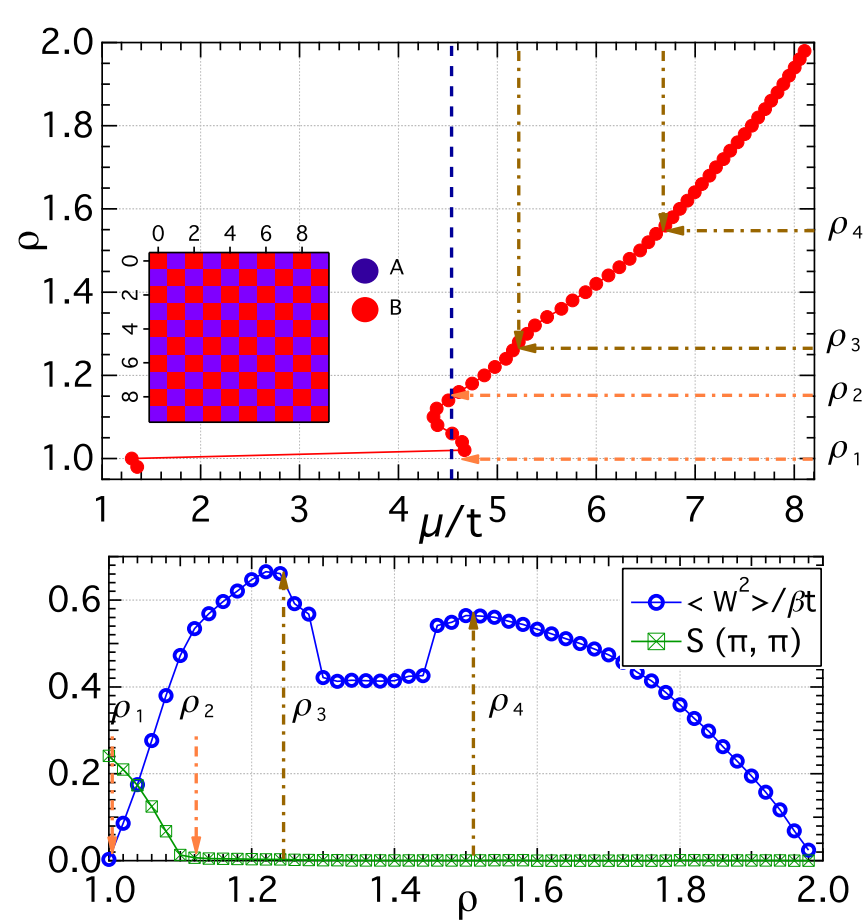

FIG. 1: (Color online) Top panel: The average density, $\rho=$ $N / L$ as a function of the chemical potential, $\mu$. The vertical blue dashed line shows the Maxwell construction between $\rho_{1}$ and $\rho_{2}$. The brown dotted-dashed lines show different phase boundaries at $\rho_{3}$ and $\rho_{4}$ as discussed in the text. The inset shows a snapshot of the density profile at half filling, $\rho=\rho_{1}=$ 1 , with the blue (red) squares indicating $\left\langle n_{i}^{a}\right\rangle=1\left(\left\langle n_{i}^{b}\right\rangle=1\right)$. Bottom panel: The correlated winding (blue circles) and the staggered structure factor (green squares) as a function of $\rho$. The dot-dashed lines show different phase boundaries. All data are for $L=10, \beta=80, t_{a}=0.08, t_{b}=1.00$ and $U^{a b}=6$. Error bars are smaller than symbol sizes.

$W_{a}$ and $W_{b}$ are the windings of particle $a$ and $b$ respectively. The AF phase is characterized by a finite densitydensity static structure factor: $S(\mathbf{k})=\frac{1}{L^{2}} \sum_{k, l} \exp [i \mathbf{k}$. $\left.\left(\mathbf{r}_{k}-\mathbf{r}_{l}\right)\right]\left\langle n_{k}^{(a, b)} n_{l}^{(a, b)}\right\rangle$. We find an AF phase at half filling $\rho_{1}=1$. It is characterized by a vanishing compressibility, $\kappa=\partial \rho / \partial \mu$ (top panel), a finite static staggered structure factor (bottom panel), as well as AF ordering as shown in a snapshot of the density profile (inset of the top panel). Near half filling, $\rho=N / L$ vs. $\mu$ displays a first-order phase transition between $\rho_{1}$ and $\rho_{2} \sim 1.16$. The instability is characterized by a region of negative slope. These two phases with densities $\rho_{1}$ and $\rho_{2}$ coexist for any density value between the two end points. Since, in this region the system displays finite values of $S(\pi, \pi)$ and correlated winding we conclude that the $\mathrm{AF}$ and $\mathrm{SF}$ phases coexist for any $\rho_{1}<\rho<\rho_{2}$. A homogeneous SF state exists between $\rho_{2}$ and $\rho_{3} \sim 1.25$ identified by measuring the correlated winding $\left\langle W^{2}\right\rangle$. At $\rho_{3}$ the superfluid density displays a decrease and the $\rho$ versus $\mu$ plot shows a small bump. Another small feature is displayed in the top panel at $\rho_{4} \sim 1.52$. Finally, the homogeneous SF phase continues until full filling. Next, we investigate the unexpected lowering of the superfluid density between $\rho_{3}$ and $\rho_{4}$.
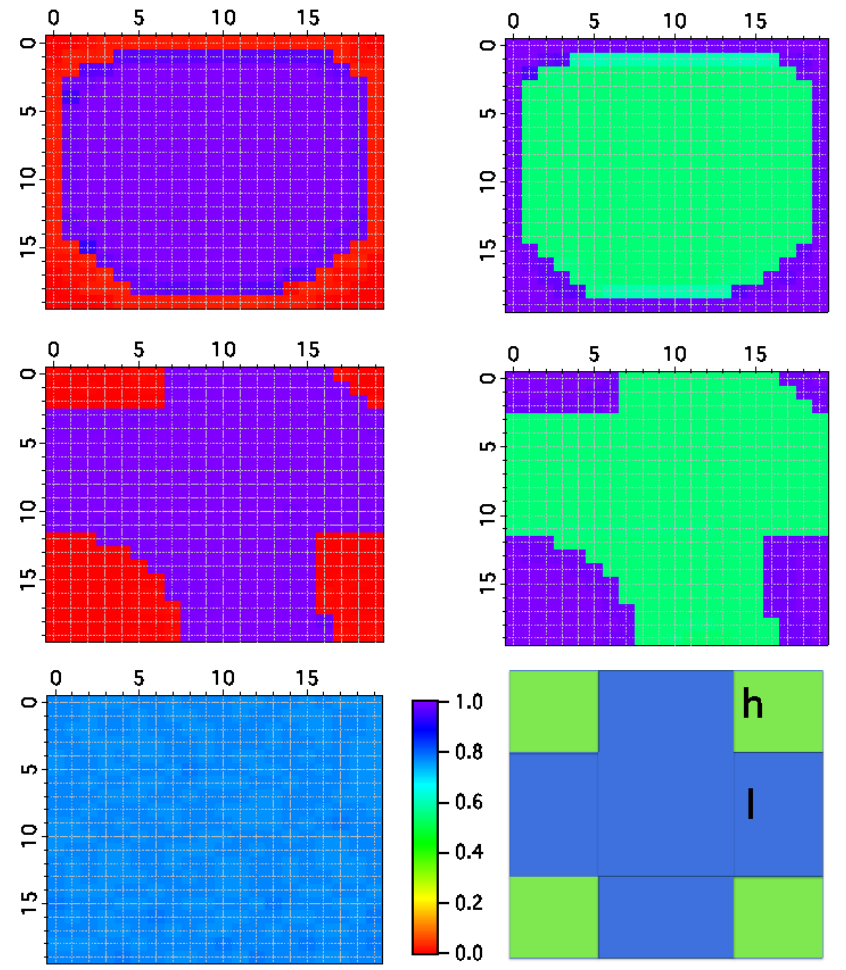

FIG. 2: (Color online) Snapshot of the average local densities for $L=20$ and $\rho=1.44$. Top panel: Open boundary conditions. For $a$ particles (left panel), sites close to the boundary (red) have $\left\langle n_{i}^{a}\right\rangle \sim 0$, while the occupation of the central (blue) region is $\left\langle n_{i}^{a}\right\rangle \sim 1$. For $b$ particles (right panel) the density close to the boundary (blue region) is $\left\langle n_{i}^{b}\right\rangle \sim 1$, and at the center (green region), $\left\langle n_{i}^{b}\right\rangle \sim 0.60$. Middle panel: The same quantities shown in the top panel but with periodic boundary conditions. Bottom panel: At the left the homogeneous density distribution of both $a$ and $b$ particles for $\rho=1.72$. At the right, a sketch of the density profile for a simulation with periodic boundary conditions.

Fig. 2 shows snapshots of the average local density of both species for $\rho_{3}<\rho=1.44<\rho_{4}$. Simulations with both open and periodic boundary conditions show clear evidence of ferromagnetic phase separation into regions with polarizations $\left\langle n_{i}^{a}-n_{i}^{b}\right\rangle$ of opposite sign. The heavy species $a$ shows Mott behavior with integer fillings, $\left\langle n_{i}^{a}\right\rangle \sim 0$ or 1 , while the light species $b$ shows Mott $\left(\left\langle n_{i}^{b}\right\rangle \sim 1\right)$ and SF $\left(\left\langle n_{i}^{b}\right\rangle \sim 0.60\right)$ phases. We can understand the tendency of the system to form such a mixed phase by extending the bosonic mean-field formalism 2,24 to two species in the hardcore limit $\stackrel{11}{=}$ We use the Gutzwiller variational approach, $\stackrel{25}{\rightleftharpoons}$ in which the most 
general site factorized wave function can be written as

$$
\begin{aligned}
\Psi=\prod_{i} \Psi_{i}= & \prod_{i}\left[\sin \frac{\theta}{2}\left(\sin \frac{\alpha}{2} a_{i}^{\dagger}+\cos \frac{\alpha}{2} b_{i}^{\dagger}\right)\right. \\
& \left.+\cos \frac{\theta}{2}\left(\sin \frac{\beta}{2}+\cos \frac{\beta}{2} a_{i}^{\dagger} b_{i}^{\dagger}\right)\right]|0\rangle,
\end{aligned}
$$

where $\theta, \alpha$ and $\beta$ are variational parameters and $|0\rangle$ is the vacuum state. The energy per site takes the form

$$
\begin{aligned}
\frac{E}{L^{2}}= & -t_{a} \sin ^{2} \theta \cos ^{2}\left(\frac{\alpha-\beta}{2}\right)-t_{b} \sin ^{2} \theta \sin ^{2}\left(\frac{\alpha+\beta}{2}\right) \\
& +U^{a b} \cos ^{2} \frac{\theta}{2} \cos ^{2} \frac{\beta}{2} .
\end{aligned}
$$

We solve these equations by minimizing the energy of the superfluid and phase separated states when $L=20, t_{a}=$ $0.08, t_{b}=1.0$ and $\rho=1.44$, subject to the constraints $n_{a}=\frac{N_{a}}{L^{2}}=\sum_{i} \frac{\left\langle\Psi\left|n_{i}^{a}\right| \Psi\right\rangle}{L^{2}}$ and $n_{b}=\frac{N_{b}}{L^{2}}=\sum_{i} \frac{\left\langle\Psi\left|n_{i}^{b}\right| \Psi\right\rangle}{L^{2}}$. For $\rho=1.44$ we find that the phase separated state is lower in energy than a homogeneous superfluid phase. The total energy of a homogeneous superfluid with densities $\rho_{a}=\rho_{b}=0.72$ is 804.33 . The phase separated state is illustrated in the bottom right panel of Fig. 2 with a central cross with area $(2 h+l)^{2}-4 h^{2}$ and four corner squares of area $h^{2}$. If we assume that in the cross $\left\langle n_{i}^{a}\right\rangle=1.0$ and $\left\langle n_{i}^{b}\right\rangle=0.60$ for all $i$ sites, then the meanfield energy is 754.19 . The PS region is stabilized by the reduction of the potential energy, consequently there is a critical $U_{c}^{a b}$ above which the phase separated state is stable. We estimate $U_{c}^{a b} \sim 4.8$.

Next we study the temperature dependence of $\rho$ and $\rho_{S F}$ using the correlated winding. The top panel of Fig. 3 shows $\rho$ versus $\mu$ for a system of size $L=10$ and different inverse temperatures. Since there is a clear signature of phase separation for $\beta=6.5$ but not for $\beta=1.5$, we can estimate that the critical AF temperature occurs between these two temperatures. Similarly, from the $\left\langle W^{2}\right\rangle$ vs. $\rho$ curves for different temperatures (see bottom panel of Fig. 31), we can conclude that for this cluster size the phase separated region between $\rho_{3}$ and $\rho_{4}$ appears for temperatures between $\beta=6.5$ and $\beta=12$. We infer the phase diagram by appropriate scaling of our finite-size results.

Fig. 4 displays the temperature, $T$, vs. doping, $\delta=$ $\frac{N_{a}+N_{b}}{2 L^{2}}-\frac{1}{2}$, phase diagram. In the thermodynamic limit, the $\mathrm{AF}$ phase only exists at half filling $\delta_{1}=0$ and low temperatures. The top left panel shows the scaling of the AF to normal liquid (NL) continuous phase transition at $\delta_{1}=0$. This transition belongs to the two-dimensional Ising universality class for which the static staggered structure factor scales as $S(\pi, \pi)=$ $L^{-(2 \beta / \nu)} f\left(\left(T-T_{c}^{A F}\right) L^{1 / \nu}\right)$, where $f$ is a universal scaling function, and $\beta$ and $\nu$ are the critical exponents for the order parameter and the correlation length, respectively. The factor $2 \beta / \nu=1 / 4$ in two-dimensional systems. Therefore we can read the critical temperature at
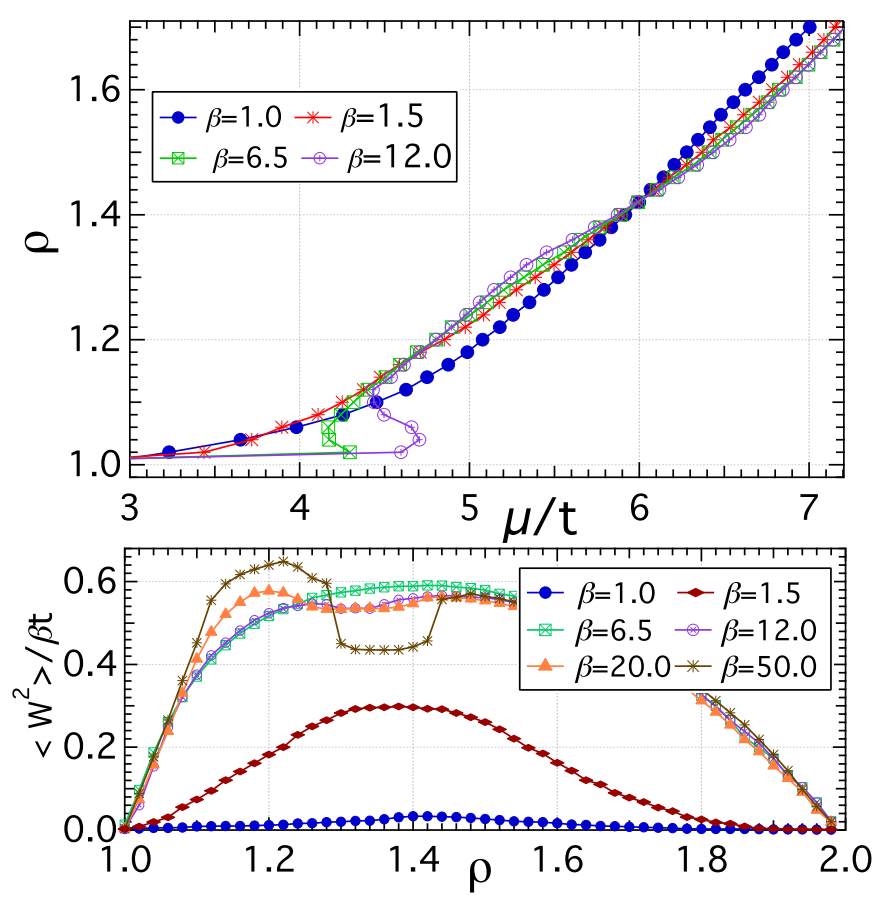

FIG. 3: (Color online) Top panel: Average density, $\rho$, versus chemical potential, $\mu$, for different temperatures. Bottom panel: The correlated winding as a function of $\rho$ for different temperatures. All data are for system size $L=10$. Error bars are smaller than symbol sizes.

the point where the $S(\pi, \pi) L^{1 / 4}$ vs. $T$ curves for different system sizes cross. For our parameters $T_{c}^{A F}=0.116$, and $S(\pi, \pi) L^{1 / 4}$ vs. $\left(T-T_{c}^{A F}\right) L$ curves collapse. The $\mathrm{AF}$ phase is represented by a red line ending on a blue diamond in Fig. 4. As we illustrate in previous figures, near half filling, we find a discontinuous transition from $\mathrm{AF}$ to $\mathrm{SF}$ phases and a phase separation region for doping $\delta_{1}=0.0 \lesssim \delta \lesssim \delta_{2}=0.06$ (dark blue region in Fig. (4). The boundary of the AF/SF phase separated region is found by a Maxwell's construction of the $\rho$ vs. $\mu$ plots. The PS region inside the SF phase exists for $\delta_{3}=0.13 \lesssim \delta \lesssim \delta_{4}=0.28$. For a given temperature we determine its boundaries by estimating the filling where $\rho_{S F}$ starts decreasing ( $\rho_{3}$ in Fig. 1) or stops increasing $\left(\rho_{4}\right)$. For the rest of the dopings we encounter a SF phase at low temperatures and an NL at higher temperatures. The top right panel of Fig. [4 shows the correlated winding as a function of temperature for different system sizes. The order parameter, the superfluid density, has the universal jump of $\left\langle W^{2}\right\rangle=\frac{4}{\pi}$ at the critical point, $T_{c}(L) \underline{26}$ The transition from SF to NL belongs to the Kosterlitz Thouless universality class. We find $T_{c}$ in the thermodynamic limit by using the relation between the crossing temperature for different system sizes $T_{c}(L)$ and the cluster size: $T_{c}(L)-T_{c}(\infty) \propto \frac{1}{l n^{2}(L)}, \frac{27}{2}$ The inset on the right top panel displays this scaling. For $\delta=0.07$ 


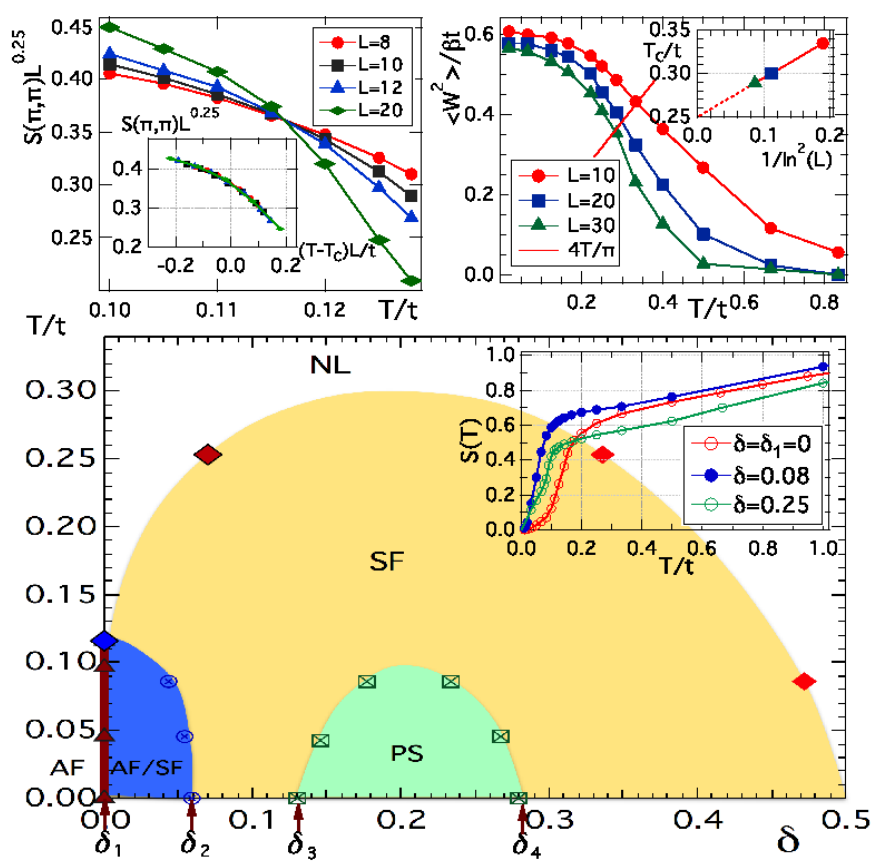

FIG. 4: (Color online) Bottom panel: The temperature, $T$, versus doping, $\delta$, phase diagram with equal population of each species. At half-filling, the system is antiferromagnetic (AF) below $T_{c}^{A F} \sim 0.116$ and normal liquid (NL) at larger temperatures. By increasing the doping a discontinuous transition from AF to superfluid (SF) phase occurs, and an AF/SF phase separated region develops between $\delta_{1}=0$ and $\delta_{2}=0.06$. Between $\delta_{3}=0.13$ and $\delta_{4}=0.28$ phase separation (PS) occurs with the heavy species becoming Mott while the light one displays regions with either Mott or superfluid behaviors. The inset shows entropy as a function of temperature for three dopings. Top left panel: Scaling behavior of the static staggered structure factor for the continuous transition from AF to NL at half filling, $\delta_{1}=0$ (corresponds to the filled blue diamond in the bottom panel). The inset shows the scaling near the critical temperature with Ising-like critical exponents. Top right panel: Correlated winding as a function of temperature for different system sizes at $\delta=0.07$ (filled red diamond). The inset shows the finite size scaling to find the $\mathrm{SF}$ critical temperature in the thermodynamic limit for the continuous transition at $\delta=0.07$. The data points are based on simulation results, the lines are guides to the eye.

( $\rho=1.14$ ) we find $T_{c}=0.25$. Scaled transition points are shown as red diamonds in Fig. 4. The inset of the bottom panel shows the entropy for a $L=10$ system calculated by following Ref. 28 for $\delta=0, \delta=0.08$ and $\delta=0.25$. The entropy of the PS ferromagnetic phase is greater than the AF phase and similar to the SF state, especially for low temperatures, indicating that it may be experimentally accessible. In addition, the entropy of this phase will mainly be carried by the light superfluid particles, enabling entropy squeezing. 20

In summary, with doping we find complex phases in the two-dimensional two-species hardcore bosonic Hubbard model for equal populations and unequal masses. We find a first order phase transition between the AF phase at half filling and a SF phase near half filling with a region of $\mathrm{SF}$ and $\mathrm{AF}$ coexistence. For a broad region of temperatures and fillings away from half, a SF phase is found. Most significantly, within the SF phase at finite doping we find a dome-shaped region containing an inhomogeneous ferromagnetic phase. Density profiles of this novel phase separated region show that the heavy species displays Mott insulating behavior, while the light species are phase separated into Mott and superfluid regions. Despite the magnetic order, this phase has an entropy much greater than the AF phase, and similar to the SF phase. For a large system size, the entropy of the heavy species in this phase is essentially zero. This phenomenon can be considered as squeezing out the entropy from the heavy species into the light species, while both species are bosonic, in contrast with the recent proposal for cooling the boson-fermion mixture ${ }^{20}$ Farther from half filling, a SF phase appears in a broad region of fillings at low temperature, while a NL phase appears at all fillings and high temperature. Further investigation of the phase diagram shows a rather complex SF phase which we discuss in future publications.

This complex phase diagram reminds us of the phase diagram of cuprates. In particular, our phase diagram displays a region that is similar to the so-called "superconducting dome" away from half-filling. Further, we believe our work will encourage experimental studies of this model on cold atoms traps. Indeed the experimental realization of the half-filling AF phase is difficult due to the low entropy associated with this phase, while the complex ferromagnetic phase that we identify away from half-filling can be expected to have a high entropy and, then, be easier to obtain. To further explore this complex phase diagram we are planning to extend our simulations to polarized systems with different population for each specie.

We thank D. Browne and D. Galanakis for useful discussions. This work is supported by NSF OISE-0952300 (KH, VGR and JM). Additional support was provided by DOE SciDAC grant DE-FC02-06ER25792 (KMT and MJ). This work used the Extreme Science and Engineering Discovery Environment (XSEDE), which is supported by the National Science Foundation grant number DMR100007, and the high performance computational resources provided by the Louisiana Optical Network Initiative (http://www.loni.org).
1 M. Greiner, O. Mandel, T. Esslinger, T. W. Hänsch, and I. Bloch, Nature (London), 415, 39 (2002).
${ }^{2}$ M. P. A. Fisher, P. B. Weichman, G. Grinstein, and D. S. 
Fisher, Phys. Rev. B 40, 546 (1989).

${ }^{3}$ G. G. Batrouni and R. T. Scalettar, Phys. Rev. Lett. 84, 1599 (2000).

4 E. Timmermans, P. Tommasini, M. Hussein, A. Kerman, Phys. Rep. 315, 199, (1999).

5 T. Köhler, K. Góral, and P. S. Julienne, Rev. Mod. Phys. 78, 1311, (2006).

${ }^{6}$ F. Schreck, L. Khaykovich, K. L. Corwin, G. Ferrari, T. Bourdel, J. Cubizolles, and C. Salomon, Phys. Rev. Lett. 87, 080403 (2001); A. Albus, F. Illuminati, and J. Eisert, Phys. Rev. A 68, 023606 (2003); G. Modugno, L. Khaykovich, K. L. Corwin, G. Ferrari, T. Bourdel, J. Cubizolles, and C. Salomon, Science, 297 (2002); C. Ospelkaus, S. Ospelkaus, K. Sengstock, and K. Bongs, Phys. Rev. Lett. 96, 020401 (2006).

7 G. Roati, M. Zaccanti, C. DErrico, J. Catani, M. Modugno, A. Simoni, M. Inguscio, and G. Modugno, Phys. Rev. Lett. 99, 010403 (2007); G. Thalhammer, G. Barontini, L. De Sarlo, J. Catani, F. Minardi, and M. Inguscio, Phys. Rev. Lett. 100, 210402 (2008); S. B. Papp, J. M. Pino, and C. E. Wieman, Phys. Rev. Lett. 101, 040402 (2008).

8 J. Catani, L. De. Sarlo, G. Barontini, F. Minardi, and M. Inguscio, Phys. Rev. A 77, 011603 (2008).

9 M. Taglieber, A.-C. Voigt, T. Aoki, T. W. Hänsch, and K. Dieckmann, Phys. Rev. Lett. 100, 010401 (2008).

10 S. Taie, Y. Takasu, S. Sugawa, R. Yamazaki, T. Tsujimoto, R. Murakami, and Y. Takahashi, Phys. Rev. Lett. 105, 190401 (2010).

11 E. Altman, W. Hofstetter, E. Demler, and M. D. Lukin, New J. Phys. 5, 113 (2003).

12 S. G. Söyler, B. Capogrosso-Sansone, N. V. Prokof'ev, and B. V. Svistunov, New J. Phys. 11, 073036 (2009); B. Capogrosso-Sansone, S. G. Söyler, N. V. Prokof'ev, and B. V. Svistunov, Phys. Rev. A 81, 053622 (2010).
13 S. Powell, Phys. Rev. A 79, 053614 (2009).

14 A. Sotnikov, D. Cocks, and W. Hofstetter, Phys. Rev. Lett. 109, 065301 (2012).

15 E. Khatami and M. Rigol, Phys. Rev. A 86, 023633 (2012).

16 A. Moreo, S. Yunoki, and E. Dagotto, Science, 283, 2034 (1999); M. Uehara, S. Mori, C. H. Chen, and S.-W. Cheong, Nature 399, 560 (1999); E. Dagotto,T. Hotta, and A. Moreo, Phys. Rep. 344, 1 (2001); P. Coleman and A. J. Schfield, Nature, 433, 227 (2005); E. Dagotto, Science, 309, 257 (2005).

17 C. Monroe, D. M. Meekhof, B. E. King, S. R. Jefferts, W. M. Itano, D. J. Wineland, and P. Gould, Phys. Rev. Lett. 75, 4011 (1995).

18 M. Popp, J.-J. Garcia-Ripoll, K. G. Vollbrecht, and J. I. Cirac, Phys. Rev. A 74, 013622 (2006).

19 X. Li, T. A. Corcovilos, Y. Wang, and D. S. Weiss, Phys. Rev. Lett. 108, 103001 (2012).

20 T.-L. Ho and Q. Zhou, Proc. Natl. Acad. Sci. USA 106, 6916 (2009).

21 V.G. Rousseau, Phys. Rev. E 77, 056705 (2008); V. G. Rousseau, Phys. Rev. E 78, 056707 (2008).

${ }^{22}$ V.G. Rousseau and D. Galanakis, arXiv:1209.0946 (2012).

23 E. L. Pollock and D. M. Ceperley, Phys. Rev. B 36, 8343 (1987).

24 K. Sheshadri, H. R. Krishnamurthy, R. Pandit and T. V. Ramakrishnan, Europhys. Lett. 22, 257 (1993).

25 D. S. Rokhsar and B. G. Kotliar, Phys. Rev. B 44, 10328 (1991).

26 D. R. Nelson and J. M. Kosterlitz, Phys. Rev. Lett. 39, 1201 (1977).

27 M. Boninsegni, and N. Prokof'ev, Phys. Rev. Lett. 95, 237204 (2005).

28 F. Werner, O. Parcollet, A. Georges, and S. R. Hassan, Phys. Rev. Lett. 95, 056401 (2005). 